\title{
Bon fils, bon mari et bon père ? Antoine-Jean Solier par lui-même (1760-1836)
}

Devoted son, husband and father? Antoine-Jean Solier by himself(1760-1836)

Sylvie Mouysset et Danielle Rives

\section{OpenEdition}

Journals

Édition électronique

URL : https://journals.openedition.org/clio/10282

DOI : 10.4000/clio.10282

ISSN : 1777-5299

Éditeur

Belin

Édition imprimée

Date de publication : 31 décembre 2011

Pagination : 137-152

ISBN : 978-2-8107-0170-4

ISSN : 1252-7017

Référence électronique

Sylvie Mouysset et Danielle Rives, «Bon fils, bon mari et bon père ? Antoine-Jean Solier par lui-même (1760-1836) », Clio. Femmes, Genre, Histoire [En ligne], 34 | 2011, mis en ligne le 31 décembre 2013, consulté le 22 avril 2022. URL : http://journals.openedition.org/clio/10282 ; DOI : https://doi.org/ 10.4000/clio.10282 


\title{
Bon fils, bon mari et bon père? Antoine-Jean Solier par lui-même (1760-1836)
}

\author{
Sylvie MOUYSSET \& Danielle RIVES
}

Le 8 septembre 1797, le négociant marseillais Antoine-Jean Solier s'adresse en ces termes à son frère Marc :

Tu sais que nous formons une nombreuse famille, je l'ai toujours soutenue jusqu'à ce moment, mais j’ai besoin que tu m'aides parce que, depuis quatre ans, je ne fais que fondre mon beurre, et si les affaires ne reprennent pas, il ne durera pas longtemps ${ }^{1}$.

Le ton est ici donné de l'esprit et de la lettre des relations entretenues par l'auteur avec sa famille. Le style est direct, sans ambages ni fioritures. Protecteur, volontiers donneur de leçons, Antoine-Jean joue sa partition préférée, celle d'aîné des Solier de Camarès qui érige la solidarité familiale en force vitale du groupe.

Au fil d'une plume bavarde, dont le crissement scande ses jours, il dessine les contours de sa vie de fils, de frère, puis d'époux et de père. Né en 1760 au Pont-de-Camarès en Rouergue, Antoine-Jean quitte les confins de sa province et sa famille de modestes marchands pour les rives de la Méditerranée d'où il se lance dans le négoce outreatlantique $^{2}$. Son caractère et son parcours singuliers forgent un

1 Lettre d'Antoine-Jean Solier, 18 J 13, «Copies de lettres », $\mathrm{f}^{\circ}$ 281, Archives Dép. de l'Aveyron [ADA].

2 Dermigny 1960 ; Mouysset 2003, 2006 et 2007 ; Rives 2010 et 2011. Tandis que Louis Dermigny a très finement étudié l'œuvre du négociant, nous côtoyons depuis bientôt dix ans l'intimité d'Antoine-Jean Solier, et ce grâce à ses écrits du for privé aussi bavards que les registres de commerce et la volumineuse correspondance professionnelle conservée aux Archives départementales de l'Aveyron. Les écrits ordinaires de Solier, dont la plus grande partie est consultable à la Société des Lettres de l'Aveyron, se déclinent en "Notes 
personnage attachant, sensible, ouvert aux autres, livrant à son cahier le moindre de ses sentiments, de joie ou de colère, de déception ou d'enthousiasme. À travers sa correspondance, ses notes et son œuvre principale intitulée « Testament olographe d'un bon père à l'intention de ses enfants Bien aimés » et rédigée tout au long de sa vie à partir de la naissance de son premier enfant, il dévoile ses affects, sans interdit ni fausse honte 3 . Toujours pudique cependant, Solier noue par écrit des liens forts au sein de sa grande famille. Des souvenirs de son enfance choyée de "premier de couvée » à l'expression de son chagrin lors de la disparition de l'un des siens, se dessinent les contours de l'affectivité complexe d'un homme des Lumières. Son discours s'enracine dans la morale traditionnelle tout en annonçant les prémisses de la sensibilité romantique. Edward Shorter analyse fort bien l'irruption du «concept de sentiment» dans les relations entre individus ${ }^{4}$; le milieu du XVIII ${ }^{e}$ siècle constitue une charnière décisive dans cette mutation sociologique, le surgissement de liens affectifs inédits contribuant à «cimenter la famille conjugale » au détriment des relations traditionnelles avec le monde extérieur ${ }^{5}$.

Sentiment et émotion sont exprimés ici à travers les tendresses du fils et du père, l'attachement filial puis paternel d'Antoine-Jean constituant le fil continu de sa vie. Il en est de même des attentions délicates de l'époux ou de l'ami, soucieux d'honorer en l'autre sa spécificité et sa liberté. Pourtant, les exigences d'un caractère entier, épris de justice et d'ordre au sein d'une famille dont il s'estime responsable en qualité d'aîné, ramènent toujours Antoine-Jean à la société ancienne et traditionnelle dont il est issu. L'homme de devoir, attaché au respect des principes et d'une morale plus civique que religieuse, côtoie ainsi l'être sensible au cœur débordant d'affection, soucieux de la santé et du bonheur des siens.

diverses", "Notes commencées à Marseille et continuées à Camarès", nombreux carnets de petit ou grand formats. Son œuvre majeure reste ce Testament olographe destiné à ses enfants, fort de 310 feuillets rédigés d'une plume dense et alerte qui court tout au long de sa vie d'époux et de père aimant, soit un peu moins de quarante ans (1801-1836), à la césure entre deux mondes, l'ancien et le moderne.

3 Rives 2010.

4 Shorter 1974

5 Ibid. : 1034. 


\section{Un bon fils...}

L'univers émotionnel des quarante premières années d'Antoine-Jean est celui d'une famille nucléaire au sens strict. Alors qu'il se contente d'allusions insignifiantes à sa vie sentimentale de jeune homme, il souligne la force et la constance des liens filiaux et fraternels. La famille occupe une "place centrale $[\ldots]$ dans la construction de l'identité individualisée » ${ }^{6}$, souligne François de Singly. En outre, au fil de sa correspondance à ses parents, Antoine-Jean manifeste très tôt le sentiment de sa mission d'aîné. La déférence rituelle exprimée à leur égard - «mon très cher père »-, n’a de limite que sa vocation de chef de meute et le désir d'imposer sa manière de conduire les affaires familiales. Ses affects sont ainsi teintés d'un vernis puritain révélateur de son éducation protestante.

\section{Des tendresses de l'enfance aux larmes de l'exil}

Au fil de son Testament olographe, Antoine-Jean réinvente les épisodes enchantés de son enfance. Profitant de son statut de premier-né - ils seront douze -, il jouit de la tendresse infinie de ses parents qui autorise toutes les fantaisies : dormir avec ses éperons au risque de lacérer draps et jambes ; jouer avec les boucles d'oreilles de sa mère et en avaler une par inadvertance... « On me gâtait si fort » ${ }^{7}$, avoue-t-il avec tendresse. Enfant choyé, Antoine-Jean se souvient de la douceur de la veillée où, depuis son lit d'enfant, il se berçait des conversations d'invités qui le cajolaient au passage. Il se souvient aussi des souris que son père attrapait gaiement et déguisait pour lui en chevaux d'équipage8. Par ailleurs, il raconte comment, grâce à l'amitié du docteur Tissot, il fut «le premier inoculé dans le Pont de Camarès »? Soigné, entouré, cajolé, sa position enviable de fils élu est lisible à chaque page, de la complicité de sa mère, sa meilleure confidente, à la «douceur et discrétion» de son père, dont il devient peu à peu le mentor tout en déplorant sa faiblesse de caractère ${ }^{10}$.

\footnotetext{
Singly 2005.

Testament olographe [TO], $\mathrm{f}^{\circ} 4$.

Ibid., $\mathrm{f}^{\circ} 6$.

Ibid., $\mathrm{f}^{\circ} 14$.

$10 \quad$ Ibid., f ${ }^{\circ} 13$ et 171 .
} 
Les exigences de sa formation de marchand l'obligent à quitter prématurément ce douillet cocon familial. Cette séparation hâte sa conscience des responsabilités dès son plus jeune âge et contribue à forger son tempérament de meneur d'hommes. Il entre donc en apprentissage et, de Saint-Affrique à Marseille, goûte les fruits amers de l'exil. Il exprime à loisir ce besoin «de connaissance et de reconnaissance» des autres, indispensable au processus de construction de son identité ${ }^{11}$ :

Je vous prie de faire en sorte qu'à l'avenir je sois moins longtemps privé de vos chères nouvelles; songez que je suis dans un pays où je n'ai plus le moindre parent et que rien ne peut me dédommager de la privation de vos lettres ${ }^{12}$.

\section{Un fils et frère aîné responsable des siens}

Son départ de Camarès signe la fin d'une enfance insouciante. En pension dès l'âge de neuf ans chez de lointains parents appartenant à la sphère protestante du négoce languedocien, il doit, outre sa formation d'écolier, rendre quelques menus services à ses hôtes. La rudesse de ses pédagogues le surprend, mais Antoine-Jean saisit promptement les nouvelles règles de sa vie sociale et s'impose sans difficulté dans un milieu où règne une réelle carence affective, ne craignant de recevoir des coups ni, si nécessaire, de les rendre. Cette initiation façonne un caractère entreprenant de nature; dans la posture du jeune migrant parti tenter sa chance ailleurs, Solier se considère, avant même d'avoir assuré sa position, comme responsable de l'avenir des siens. En octobre 1779, il n'a pas vingt ans lorsqu'il annonce: «si je suis impatient de mon avancement c'est qu'alors je pourrai travailler à une petite fortune qui ne me sera précieuse qu'autant que je pourrai vous la faire partager $»^{13}$. Plus tard, alors qu'un orage a dévasté Camarès, il déplore « l'affligeante nouvelle » et conclut, non sans lyrisme: «j'ai espérance de combler ma félicité en faisant la vôtre car c'est d'elle que dépend la mienne, et je vous le répète, sans l'idée de vous rendre heureux, la vie me serait à charge $»^{14}$.

\footnotetext{
11 Singly $2005: 11$.

12 TO, f 100.

13 Ibid., fo 84 .

14 Ibid., fo 87.
} 
Peu avare de ses deniers, l'aîné cultive généreusement l'art du don : ce sont de petits cadeaux à ses frères et sœurs, acquis grâce au pécule amassé durant son apprentissage nîmois en 1776, ou encore cinquante livres confiées à un ami de ses parents, priant ceux-ci de les " répartir entre mes frères et sœurs $»^{15}$. De même, il est soucieux du confort de ses parents dont les ressources n'assurent pas une vie décente. En l'attente d'assumer lui-même cette charge, il appelle au secours l'oncle Marc, mécène de la famille dont la générosité est à l'origine de sa propre ascension sociale. Dans une lettre à sa mère, il promet son aide: "Si j'ai quelque superflu, et que vous en ayez besoin, je satisferais mon cœur en vous les offrant $»^{16}$. Selon son bienfaiteur, un jeune homme sans fortune qui entre dans le commerce «doit se refuser tout ce qui n'est pas de nécessité indispensable [...]. Il ne doit penser qu'à se mettre à même d'aider ses frères » et se regarder "comme obligé de travailler pour soutenir sa famille puisqu'il est l'aîné $»^{17}$. La leçon a été parfaitement comprise. Solier n'entend pas pour autant limiter son rôle à celui de simple bienfaiteur. Il suit attentivement la formation de ses frères, s'enquiert de leurs progrès, surveille leur orthographe, les encourage patiemment et s'investit personnellement dans la quête du bon maitre afin de leur éviter ses propres déboires. Son sens des responsabilités va donc bien au-delà du simple soutien matériel. Il revendique aussi un certain droit de regard sur la vie des siens, n’hésitant pas à morigéner ses parents au sujet de l'établissement matrimonial de ses sœurs ou professionnel de ses frères. Sur le destin de sa fratrie, l'aîné entend être consulté, écouté, voire obéi.

En véritable chef d'orchestre, il désigne sa place à chacun de ses frères au sein de l'entreprise qu'il fonde en 1790. Tandis que Marc part aux Antilles, Guillaume est envoyé dans un comptoir des bords de la mer Rouge. Outre la trajectoire professionnelle des garçons, Solier entend également régenter la destinée des filles. Dans une lettre adressée à sa mère en 1777, il dicte sa loi : « je recommande à mes sœurs d'être bien rangées, obéissantes et laborieuses [...]. Leur

\footnotetext{
15 Ibid., f 100.

16 Ibid., $\mathrm{f}^{\circ} 99$

17 Ibid., $\mathrm{f}^{\circ} 96$.
} 
caractère doit être votre principale attention $»^{18}$. Son affection à leur égard est sincère : "si mes sœurs ont envie de quelque chose qu'il n'y ait pas chez vous, je le leur achèterai $»^{19}$. On perçoit clairement la division des tâches éducatives : à la mère incombe naturellement la formation des filles dont il convient de développer les qualités d'obéissance et de discrétion ; au père revient la mission de redresser le caractère frondeur et exalté des fils en leur indiquant le droit chemin du labeur.

Au fil de sa correspondance, l'aîné s'enquiert de la santé et des progrès de ses sœurs, et donne son avis sur toute affaire jugée essentielle. Ainsi, lorsqu'un voisin regarde l'une d'elles d'un peu trop près, il réagit vivement :

Je vous avoue que je suis inquiet de voir à quel point on pousse chés vous la fureur à se marier. [...] Je serais très fâché que mes sœurs en fussent trop éprises [...] et si elles allaient contre mes désirs et le vœu de leur famille, elles en seraient les seules victimes. Je renonce quant à moi à toute idée de mariage [...]. Peut-être parce que je vois que ce sacrifice est nécessaire pour que je puisse vous porter du secours, non seulement à vous, mais à tous mes frères et sœurs; il est naturel que je doive attendre de leur reconnaissance [...]. C'est à vous mon cher Père à les y entretenir par vos bonnes exhortations que leur âge rend indispensable ${ }^{20}$.

Cette mise en demeure s'achève par la menace de ne « jamais remettre les pieds à Camarès » si l'une « d'elles se comportât mal » ${ }^{21}$. La porte presque claquée, le rapport de force institué par le rang dans la fratrie se double d'un rapport de genre ici. La domination paternelle est, en effet, sollicitée en renfort de la volonté du frère qui s'impose comme arbitre masculin de l'avenir matrimonial des filles de la famille, ses sœurs, invitées à se conformer «à nos désirs» et à contraindre «leur cœur $»^{22}$. Dans cet examen des relations horizontales à l'intérieur de la fratrie, rivalités et conflits aiguisent le discours, mais aussi affection et solidarités ${ }^{23}$. Les rapports entre aîné et cadets, garçons et filles, émergent naturellement de

\footnotetext{
18 Ibid, $\mathrm{f}^{\circ} 45$.

19 Ibid., fo 44

20 Ibid., fo 110-111.

21 Ibid., $\mathrm{f}^{\circ} 111$.

22 Ibid.

23 Fine 2007.
} 
ces écrits, rappelant à la fois la hiérarchie des âges et des sexes. Par ailleurs, le comportement autoritaire d'Antoine-Jean participe sans aucun doute de la volonté d'accomplissement de soi qui « ne se construit que par le détour de la communication extérieure avec des individus proches, des autruis significatifs $»^{24}$.

Or, pour atteindre cette connaissance/reconnaissance, les relations personnelles et affectives sont requises la vie durant, sollicitant «en permanence les liens avec les plus proches». Au cœur d'une relation de confiance confortée par amour et affection, la famille permet cette «révélation de soi » ${ }^{25}$. Dans cette quête se construit une double relation qui regarde les parents d'une part, le conjoint et les enfants de l'autre, à des échelles et dans des temporalités différentes. Antoine-Jean, la quarantaine atteinte, bouleverse son horizon et son mode de vie en se mariant au pays et en s'y enracinant comme rentier de la terre. Le célibataire vertueux et dévoué à ses pères et frères se métamorphose alors en époux et père comblé, soucieux d'assurer à sa descendance les conditions matérielles et morales d'une vie harmonieuse.

\section{" Bon mari » et " nouveau père "}

Solier convole en 1800, période de grands bouleversements normatifs des rapports intrafamiliaux ${ }^{26}$. Grand admirateur de Rousseau, mais aussi des moralistes du Siècle des Lumières, ses affects de père et d'époux sont fortement influencés par le fruit de ses lectures et de ses réflexions.

\section{Du mariage arrangé à l'harmonie du couple}

L'annonce des épousailles s'inscrit dans la plus pure tradition d'Ancien Régime. Toute la famille est mobilisée pour la quête de la compagne idéale :

Ma sœur Rose Montet de Milhau vint me voir, ainsy que mon cousin Mazarin de St-Affrique, etant à nous chaufer vers le milieu de frimaire, ils

\footnotetext{
24 Singly $2005: 12$.

25 Ibid. : 13.

26 Verjus 2010.
} 
me parlèrent de mariage et entr'autres partis ils m'indiquèrent Mlle Agathe Dalaret ${ }^{27}$.

L'élue est enfin repérée. Antoine-Jean invente un prétexte pour la rencontrer chez ses parents et, "l'extérieur m'ayant convenu, je chargeai ma sœur de me savoir dire si cette demoiselle était libre $»^{28}$. L'accueil étant favorable, il dicte à son père une lettre édifiante de demande en mariage à l'intention de son futur beau-père ${ }^{29}$. L'affaire est rondement menée et l'hymen scellé en moins d'un mois.

L'idéal du bon mariage selon Antoine-Jean est en tous points conforme au modèle traditionnel. Dans ses conseils à ses enfants, il dénonce les unions précoces ou tardives. On ne doit pas «se marier uniquement pour soi, il faut songer à ses enfants $»^{30}$, le mariage ayant pour fonction essentielle d'assurer la descendance. Les effets d'une jeunesse dissipée sont opposés aux vertus de la sienne, " passée dans l'innocence, une santé sagement ménagée, une chaste union entre les personnes qui s'aiment, un esprit cultivé et nourri de bons principes, un cœur libre de passions violentes ${ }^{31}$. Son panthéon littéraire est alors convoqué : selon Rousseau, «l'amour n'est pas toujours nécessaire pour former un heureux mariage, l'honnêteté, la vertu et certaines convenances moins de condition et d'âge que de caractère et d'humeur suffisent entre deux époux $»^{32}$. Antoine-Jean, tel le Lusane du Bon mari de Marmontel, est "plus recueilli, plus assidu» et son "Amélie » est une Agathe qui "ne vivait que pour lui», «le soin mutuel de se complaire» retenant toute leur attention ${ }^{33}$. Cette bourgeoise vision du couple dénonce, comme chez Marmontel, les illusions de la frivolité et des relations superficielles au profit «d'une communauté d'intérêts, d'amis et de mœurs $»^{34}$. Le lien qui attache l'homme à son épouse, «qui peut n'être pas précisément de l'amour,

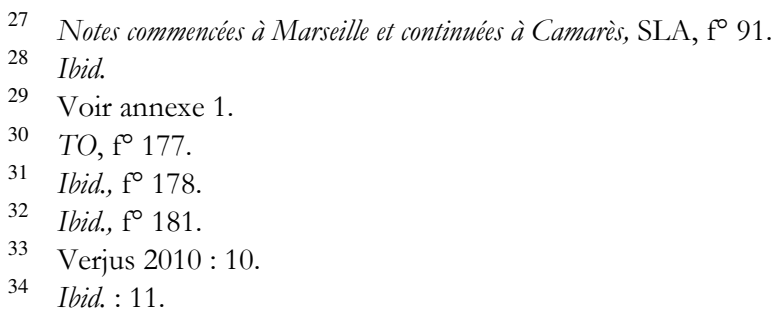


n'en est pas moins doux, et n'en est que plus durable $»^{35}$. AntoineJean épilogue : «On ne s'épouse pas pour penser uniquement l'un à l'autre, mais pour remplir conjointement les devoirs de la vie civile, gouverner prudemment sa maison, bien élever ses enfants $»^{36}$.

Cette vision classique du mariage voisine cependant avec une idéalisation très moderne de sa vie de couple. Loin de considérer ses relations avec Agathe en termes d'autorité, il semble avoir assimilé le nouveau credo du parfait époux : «Il faut être le compagnon et non le maître de sa femme » selon le Dictionnaire comique de Le Roux publié en $1786^{37}$. Ainsi Solier décide-t-il d'accoler le nom de son épouse au sien - et de signer désormais Solier-Dalaret - non pour en tirer profit, car il n'est pas plus prestigieux que le sien, mais afin que le patronyme « ne se perde pas $»^{38}$. Il redouble cette précaution en prénommant son deuxième fils Dalaret.

Au-delà des signes d'identité, Solier envisage sa mission éducative à parité avec Agathe et souhaite consacrer autant de temps que celleci aux soins, aux jeux et à l'éveil de ses enfants.

\section{Un père attentionné, aimant et exigeant}

Antoine-Jean ne cesse de clamer son bonheur d'être père. Il débute son Testament par un trait de tendresse dont sa plume est coutumière : "Vous vous direz: en faisant cet ouvrage le bon Papa pensait à nous $»^{39}$. Citant Racine fils, il poursuit: " $\mathrm{Si}$ chèrement aimés, ces nouriçons un jour, aux fils qui naitront d'eux rendront le même amour $»^{40}$. Cette tendresse n'a rien d'exceptionnel, comme l'a démontré la riche historiographie consacrée aux pères et à la paternité ${ }^{1}$. Antoine-Jean sème son amour paternel au fil des pages. Plus habile à évaluer ses bénéfices qu’à dépeindre ses sentiments, son lyrisme est discret, mais l'émotion intacte : "J'ai pris bien des

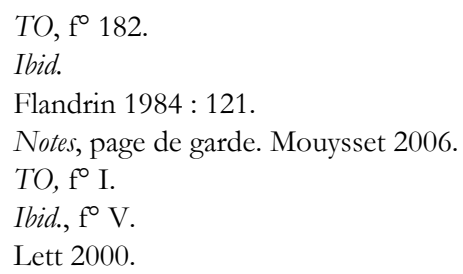


précautions pour que votre naissance fut sans accident, vous en pourrez lire le détail dans les manuscrits dont je vous ai déjà parlé $»^{42}$. À chaque naissance, il note soigneusement le moment où l'on envoie quérir la sage-femme et les conditions de l'accouchement; notes sobres, mais révélatrices de l'attention qu'il accorde à ce qui fait, de son propre aveu, sa raison de vivre ${ }^{43}$. Comptable des menus faits et gestes de ses enfants, il inscrit les dates de sevrage, celles des premières dents, des plus légères indispositions ${ }^{44}$ aux expériences plus risquées de l'inoculation de la variole ${ }^{45}$. Il se préoccupe aussi de l'avenir de sa progéniture, désireux «de vous éviter les peines par lesquelles je suis passé et de vous procurer non des jouissances continuelles, il n'y en a pas sur terre ; mais des plaisirs sans troubles $»^{46}$.

Du plaisir, Antoine-Jean en donne beaucoup à ses enfants par sa présence attentive. Appartenant à ces nouveaux pères issus des Lumières, il a lu Voltaire et préfère "une paternité douce » à celle, despotique, dénoncée par le philosophe ${ }^{47}$. Infatigable compagnon de jeu, il accueille volontiers sa joyeuse marmaille dans la «chambre boisée » qui lui sert de bureau, feuillette les livres d'images, donne les premiers rudiments de lecture, organise des séances de lanterne magique, et s'émerveille ainsi de l'émerveillement de l'enfance ${ }^{48}$. Solier entraîne ses trois garçons - mais pas ses filles - sur les sentiers de la chasse, s’ingénie à dénicher «quelques livres, quelques machines, quelques instrumens pour exciter [leur] goût», consent à leur faire «tâter » celui de la bière au retour de leurs randonnées ${ }^{49}$. La leçon de choses vaut mieux que l'étude livresque; ce nouveau père agit «comme un homme des Lumières, éveilleur d'intelligence et dispensateur du savoir $»^{50}$.

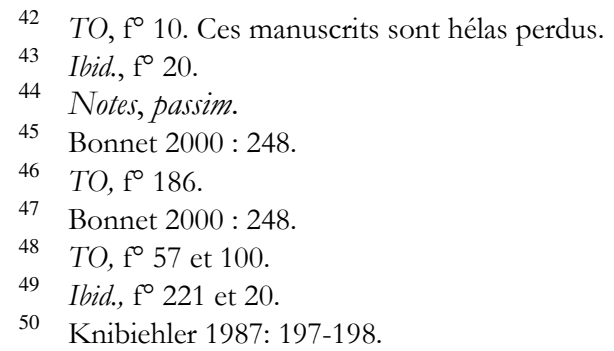


Père affectueux et exigeant, Antoine-Jean met aussi ses "petits drôles » en garde contre d'éventuelles bêtises ${ }^{51}$. Afin de leur prodiguer l'instruction qui lui a fait défaut, il se plonge dans la lecture des « systèmes d'éducation » et professe à l'égard de L'Émile une dévotion quasi religieuse. "Tout enseignement qui procède de la démonstration est celui qui grave mieux dans la mémoire les préceptes qu'il donne $»^{52}$, affirme-t-il, sensible aux débats contemporains sur les bienfaits d'une formation complète et bien pensée ${ }^{53}$.

L'intérêt de votre éducation phisique m'engage plus que tout autre motif à faire mon séjour à la campagne, et j’y employe mes veilles à vous transmettre par écrit tout ce que je crois capable d'aider à votre bonheur, afin que si je ne puis vous l'enseigner pendant ma vie, vous puissiez en profiter après ma mort ${ }^{54}$.

Inculquer le respect de valeurs morales fondamentales et ne pas céder à «leurs petits caprices », telle est l'ambition de ce père attentif :

Si vous ne deviez pas répondre à mon attente, je vous dirais comme Ulisse à Télémaque : «Oh! Mes fils que les ciseaux de la Parque tranche le fil de vos jours si vous devez vous corrompre et abandonner la vertu $»^{55}$.

Ouvert, tolérant et didactique, Antoine-Jean dresse la liste des normes sociales à ne pas enfreindre, et celle des règles domestiques indispensables au bon gouvernement de la maison ${ }^{56}$, le tout ponctué de recommandations et de formules édifiantes : «les plaisirs des sens nous donnent quelques momens agréables; ceux de la vertu nous laissent des souvenirs délicieux $\aleph^{57}$. Dans un raccourci significatif, il livre sa conception de l'existence :

L'amour pour nos parens, pour le travail, pour l'ordre, l'amitié, la bienveillance universelle, la reconnoissance sont des sentimens dont nous ne devons point nous départir ${ }^{58}$.

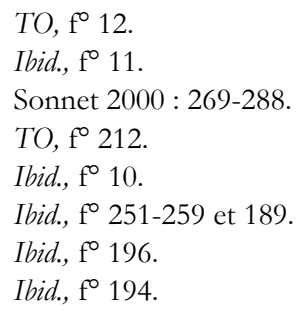


Son idéal affectif est à la fois le fruit de la morale calviniste de son enfance et de l'imprégnation forte de ses lectures moralisantes. Dans sa relation à ses enfants, soulignons cependant la spécificité du lien à ses fils. Le Testament olographe leur est d'abord adressé, tandis que l'attachement à ses filles est d'une autre étoffe.

Père et fils, père et filles

L'attitude d'Antoine-Jean est ambiguë. L'énoncé de sa tendresse ne souffre, en apparence, aucune distinction; dans les soins prodigués, dans les jeux de l'enfance, son cœur de père s'épanche avec la même émotion. Filles et garçons ne sont pourtant pas équitablement traités. Alors qu'il recherche pour ses fils une instruction rigoureuse, les inscrivant tous trois, à peine sortis de l'enfance, au collège de Sorèze - établissement réputé pour sa pédagogie novatrice-, aucune mention ne concerne l'éducation des filles. La relation apparaît ici plus affective qu'intellectuelle. L'explosion des sentiments leur est destinée en priorité selon Yvonne Knibiehler pour qui les pères, sensibles à leurs grâces et à leurs faiblesses, se soucient de les protéger en négociant un bon mariage ${ }^{59}$. Si Antoine-Jean reste muet sur les circonstances de l'union de ses filles, il leur assure un parti fort honnête, se montre soucieux de leur santé et suit de près leurs grossesses, au moins celles de son aînée. Agathe porte le nom de son épouse, fille de prédilection maintes fois citée, alors que les deux autres ne figurent au livre qu'à l'occasion de leur naissance, leur sevrage, leur inoculation, ou lors de quelque déplacement familial. Victoire-Agathe voit le jour en 1805, juste après les trois garçons; première fille, chère à son cœur, il l'entoure de ses soins, lui faisant suivre, par exemple, les leçons d'un maittre de piano ${ }^{60}$. Mariée à un magistrat, Agathe s'installe dans le berceau rouergat de la famille et devient la correspondante privilégiée de son père reparti à Marseille. Ce cordon épistolaire permet à Antoine-Jean de rêver à son cher pays. En 1829, elle offre à ce grand-père sevré de descendance un petit-fils, « énorme, œil noir, rond, double menton ressemblant à Elmence ». Quant à Dalaret, seul fils non célibataire, il garde au loin, en

$\begin{array}{ll}59 & \text { Knibiehler } 1987: 198-201 . \\ 60 & \text { Notes, fo } 184 .\end{array}$ 
Guadeloupe, sa nombreuse famille ${ }^{61}$. Cette proximité père/fille participe clairement du système éducatif dans lequel le père protecteur jouit pleinement de l'estime admirative de sa fille; leur relation s'établit dans «une sorte d'idylle $»^{62}$. Telles Manon Phlipon, future madame Roland, ou Félicité du Crest, comtesse de Genlis, Agathe a bénéficié des bienfaits d'un père attentif à son éducation ${ }^{63}$.

Le rapport père/fils est, en revanche, beaucoup plus viril et l'affection a ici plus de mal à filtrer ${ }^{64}$. L'amour paternel de Solier s'exprime discrètement; on le perçoit à travers la mélancolie de l'absence - « il y a neuf ans que Dalaret partit»-, l'importance attribuée aux lettres de l'exilé ou l'inquiétude suscitée par le défaut de nouvelles. Les préoccupations sont d'un autre ordre. Ainsi, les débuts pianistiques d'Agathe correspondent aux " principes de littérature $»^{65}$ infligés à son aîné Désiré par Mr Bayle, maître de français. Les garçons sont initiés au latin, fréquentent l'école de Sorèze, berceau de l'élite négociante. Solier tente toutefois de se persuader qu'il n'a pas de projet préconçu pour sa progéniture, n'étant pas de ces «pères qui s'imaginent que leurs goûts seront ceux de leurs enfans $»^{66}$.

Solier n'est pas un être de solitude, loin s'en faut. Sa famille est nécessaire à son existence, il n'est qu'en elle. À son image, il la dessine ample, dans un ordre qui lui est singulier, dilatée sur le monde. «En allant au devant des autres, c'est en avant de soi-même que l'on s'élance ", souligne judicieusement Anne Vincent-Buffault ${ }^{67}$. Il serait trop long ici d'évoquer la famille imaginée du négociant marseillais, un entourage élargi à ses amis de cœur. Dans les notes ultimes dictées au soir de sa vie, il égrène le nom des siens en forme d'inventairesouvenir : voici venu le temps de la nostalgie. Bien avant, la vitalité des liens noués résonne telle une basse continue dans l'ensemble de

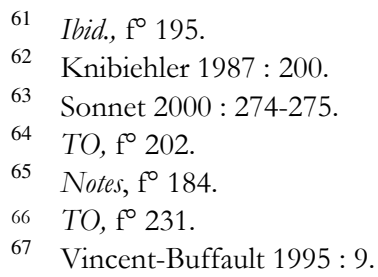


son œuvre autobiographique et jusque dans sa correspondance commerciale. Visites de voisinage, demande de nouvelles, invitations et retrouvailles, services offerts et reçus, accueil des voyageurs de passage expriment le bonheur de l'entre-soi. Son sens de la solidarité lui vaut la reconnaissance infinie de ses amis, comme de celui-ci dont les mots de remerciement l'ont ému: «homme sensible reçois les actions de grâce d'un malheureux ami qui te doit plus que la vie $\aleph^{68}$.

Solier est aussi un être fidèle. Sa relation à l'oncle Marc, cadet de l'influente branche de Saint-Félix ${ }^{69}$, est exemplaire de sa gratitude. Il évoque ce parent éloigné avec déférence - mon bon Oncle, ce cher Oncle - et dresse le portrait bienveillant «d'un homme simple et d'une aménité extrême $»^{70}$. Antoine-Jean passe à Vevey de longues semaines auprès de ce parent vieillissant et d'autant plus sensible à l'amitié du jeune homme. L'annonce de sa mort, "nous perdîmes $\mathrm{Mr}$ Marc Solier notre respectable bienfaiteur en juin $1789 \gg^{71}$, traduit l'estime et la reconnaissance sans faille témoignées par l'aîné d'une branche cousine si lointaine et si proche.

Au crépuscule d'une vie bien remplie, Solier voit à son tour se déliter cette belle et grande famille, et sa plume vacillante dit l'émotion de la perte irréversible des siens. Les toutes dernières Notes, brèves mais éloquentes, expriment l'amitié, la tristesse et le désarroi du vieil homme qui voit doucement sombrer son univers familier :

$1^{\text {er }}$ février [1833] mort de Kick [...] précédée de celles de Faque, Straforello, Genouilhac voilà quatre contemporains à peu prez et de mes plus anciennes connoissances à Marseille ${ }^{72}$.

La liste allonge son ombre funeste jusqu'à ce qu'Antoine-Jean salue solennellement sa dernière année de vie et se prépare, l'âme paisible, à rejoindre les siens dans l'autre monde :

Ô ma septante sixième, adieu, tu es finie pour moi : puissé-je dans le temps de vie que Dieu m'accordera réparer le mal que j'ai fait aux autres et à moi-même pour obtenir miséricorde et grâce ${ }^{73}$.

\footnotetext{
TO, f 168.

Mouysset $2007: 214$ et sq.

Ibid., $\mathrm{f}^{\circ} 68$.

Ibid., f $\mathrm{f}^{\circ} 157$.

Notes, fo 200 .
} 


\section{Bibliographie}

Bonnet Jean-Claude, 2000, «De la famille à la patrie ", in Daniel Roche \& Jean Delumeau (dir.), Histoire des pères et de la paternité, Paris, Larousse, p. 245-268.

Dermigny Pierre, 1960, Cargaisons indiennes, Paris, SEVPEN, 2 volumes.

FINE Agnès, 2007, «Les fratries en Europe. Aperçu sur quelques orientations de recherche en anthropologie », in Michel ORIS, Guy BRUNET \& Eric WIDMER (dir.) Les fratries. Une démographie sociale de la germanité, Bern, Peter Lang, p. 47-78.

FLANDrin Jean-Louis, 1984, Familles. Parenté, maison, sexualité dans l'ancienne société, Paris, Seuil.

FoIsIL Madeleine, 2000, «En son for privé », in Daniel Roche \& Jean DeLumeAu (dir.), Histoire des pères et de la paternité, Paris, Larousse, p. 193-218.

KNIBIEHLER Yvonne, 1987, Les pères aussi ont une histoire, Paris, Hachette.

LETT Didier, 2000, "Tendres souverains. Historiographie et histoire des pères au Moyen Âge ", in Daniel Roche \& Jean Delumeau (dir.), Histoire des pères et de la paternité, Paris, Larousse, p. 17-40.

Mouysset Sylvie, 2003, «Les cahiers d'Antoine-Jean Solier, Rouergat du Nouveau Monde ", in Rouergue, carrefour d'bistoire et de nature, Toulouse, Féd. Hist. de MidiPyrénées, Société des Lettres de l'Aveyron, p. 177-186.

—, 2006, "Me suis cy soubscrit: la signature dans les écrits du for privé », La faute à Rousseau, Association pour l'Autobiographie (APA), février, n²41, p. 28-30.

—, 2007, Papiers de famille. Introduction à l'étude des livres de raison (France, XVe-XIX $X^{e}$ siècle), Rennes, Presses universitaires de Rennes.

Rives Danielle, 2010, «De Camarès à Marseille : les lieux du négociant Antoine-Jean Solier (1760-1836) », Annales du Midi, 270, t. 122, p. 229-250.

_, 2011, «Le “Testament olographe” d'Antoine-Jean Solier (1760-1836), ou l'ego histoire à l'usage de ses enfants ", in Nicole Lemaitre \& Sylvie MouysseT (dir.), Entre mémoire et histoire : écriture ordinaire et émergence de l'individu, CTHS, édition électronique (http://cths.fr/ed/edition.php?id=5327)

SINGLY François de, 2005, Le soi, le couple et la famille. La famille, un lieu essentiel de reconnaissance et de valorisation de l'identité personnelle, Paris, A. Colin.

SHORTER Edward, 1974, «Différences de classe et sentiment depuis 1750. L'exemple de la France », Annales ESC, p. 1034-1057.

SONNET Martine, 2000, "Les leçons paternelles", in Daniel RochE \& Jean Delumeau (dir.), Histoire des pères et de la paternité, Paris, Larousse, p. 269-288.

73 Ibid., f $\mathrm{f}^{\circ} 207$. 
Verjus Anne, 2010, Le bon mari. Une histoire politique des hommes et des femmes à l'époque révolutionnaire, Paris, Fayard.

Vincent-Buffault Anne, 1995, L'exercice de l'amitié. Pour une histoire des pratiques amicales aux XVIII et XIX ${ }^{e}$ siècles, Paris, Éditions du Seuil, coll. « La couleur de la vie ».

\title{
Annexe
}

\section{Lettre dictée par Antoine-Jean Solier à son père en vue de son prochain mariage}

\author{
Notes commencées à Marseille et continuées à Camarès, f. $91-93$ \\ Société des Lettres de l'Aveyron
}

Je fis écrire par mon père la lettre suivante à Mr Dalaret.

Pont de Camarès 24 X

Mon fils ainé établi depuis 24 ans à Marseille où il a acquis honnorablement une fortune honnête et bien assise étant dans sa $39^{\mathrm{e}}$ année, possédant ici maison et campagne fort agréables, était presque décidé à remettre à ses frères qui sont à Marseille la suite de ses affaires de comerce et à se retirer ici. Ma femme et moi, persuadés que le meilleur moyen de le fixer auprès de nous, ce que nous désirions depuis longtems, était de l'y marier, ne cessions de le solliciter de se choisir une compagne. Sachant que vous aviés une aimable demoiselle a pourvoir, nous l'engageames sans lui rien dire de nos vues à vous aller faire visite, comme à un ancien ami et allié de notre famille, il revint fort enchanté d'avoir fait vôtre connoissance. Nous saisimes cette occasion pour lui renouveller nos instances. Ma fille ainée mariée chez vous, ses parens, ses amis, se sont joints à nous et il se rend enfin à nos vœux. Il est persuadé que Mlle vôtre fille Agathe est une personne de tout mérite, il croit qu'il sera heureux avec elle, en conséquence, Monsieur, je viens vous faire la demande de sa main pour mon dit fils et je désire qu'elle vous soit agréable ainsy qu'à Madame et Madlle Alaret. La médiocrité de ma fortune et de celle de ma femme ne nous permet pas de faire des avantages à nôtre dit fils, mais graces au ciel il n'en a pas besoin, il est l'auteur de sa fortune et il en sera économe. Il sera satisfait, ainsy que nous, des dons que vous jugerés à propos de faire à $\mathrm{M}^{\mathrm{lle}}$ Agathe. Il n'ambitionne que sa main et son cœur.

J'attendrai votre réponse avec l'impatience d'un octogénaire qui désire de voir son enfant heureux et de resserer les liens d'estime et de parenté qui existent entre nos deux familles.

Recevés je vous prie nos honneurs et nos cordialités et faites en part à Madame Alaret. 